\title{
Common Cause and Contextual Realization of Bell Correlation
}

\author{
A. Shafiee ${ }^{*(1)}$, R. Maleeh ${ }^{(2)}$ and M. Golshani ${ }^{(3,4)}$ \\ 1) Department of Chemistry, Sharif University of Technology, \\ P.O.Box 11365-9516, Tehran, Iran \\ 2) Department of Philosophy of Science, Sharif University of Technology, \\ P.O.Box 11365-9161, Tehran, Iran. \\ 3) Department of Physics, Sharif University of Technology, \\ P.O.Box 11365-9161, Tehran, Iran. \\ 4) Institutes for Studies in Theoretical Physics \& Mathematics, \\ P.O.Box 19395-5531, Tehran, Iran.
}

November 23, 2018

\begin{abstract}
Considering the common cause principle, we construct a localcontextual hidden-variable model for the Bohm version of EPR experiment. Our proposed model can reproduce the predictions of quantum mechanics. It can be also extended to classical examples in which similar correlations may be revealed.
\end{abstract}

\section{Introduction}

According to Bell's Theorem [1], one cannot construct a local realistic hiddenvariable theory that can reproduce all the statistical predictions of quantum mechanics for a two-particle singlet-state. Here, by the term locality we mean Bell's locality condition which in a local stochastic hidden-variable theory is equivalent to the statistical independence of the values of the spin components of the two particles [2]. Bell's locality condition at the level of hidden

*E-mail: shafiee@theory.ipm.ac.ir 
variables also implies a more general tenet named the "common cause principle" [3]. According to this principle, one can explain how two spatially separated particles can be correlated, when there is no direct causal relationship between them. Despite the fact that one cannot decisively close the door to other potential alternatives which may provide a better understanding of the so-called Bell-type correlations in a causal fashion [4], the common cause description is still a very attractive and comprehensible way of understanding the nature of these correlations [5]. But, thanks to Bell's theorem, we do know that this description cannot be compatible with the predictions of quantum mechanics. So, we are still facing the crucial problem of whether correlations resulting from the entanglement of two spatially separated particles are an essential characteristic of microphysical reality. What is then the nature of these correlations? Could they still be explained by invoking a field of common cause in the past light cones of the two separated particles?

The main purpose of this article is to give a positive answer to the last question. Here, we want to provide a stochastic hidden-variable model which honors the common cause principle but cannot be ruled out by Bell's theorem. The key point here is that it is a local contextual model. By the term contextual we mean that each probability function is defined in a measurement setup where a definite set of hidden variables is used. This approach is very similar to what has been recently suggested by de Muynck [6]. Here, we are going to provide a more concrete realization of what de Muynck has proposed as contextual reality which might be accomplished by some hidden variables in a subquantum theory.

In section 2, we first review the common cause principle in the domain of probabilistic causality. Then, in section 3, we construct a local contextual hidden-variable model which confirms the quantum mechanical predictions. In section 4, we explain why our proposed model is local and contextual. Subsequently, we talk about an alternative classical example in section 5 which can make the subtleties of our argument more transparent. We have summarized our results in the last section.

\section{Common Cause Principle}

It seems better to explain common cause principle through the term "spurious correlation". Eells defines the usual understanding of this term in the following way [7]:

"[T]wo factors are spuriously correlated when (roughly) neither causes the other and the correlation disappears when a third vari- 
able is introduced and "held fixed"- that is, the correlation disappears both in the presence and in the absence of the third factor."

If a correlation between factors $x$ and $y$ disappears both in the presence and in the absence of third factor $z$, then the explanation may be either that the correlation results from the joint causal effect of $z$ on $x$ and $y(z$ is a common cause of $x$ and $y$ ) or that $z$ is an intermediate causal factor between $x$ and $y$ ( $x$ operates on $y$ through $z$ or $y$ operates on $x$ through $z$ ). Since one of $x$ and $y$ may in fact be a genuine positive causal factor for the other in the second case, we do not consider it as a case of spurious correlation.

So, as it is indicated by Eells, two factors $x$ and $y$ are spuriously correlated if neither causes the other and the correlation of $y$ with $x$ disappears when $z$ is held fixed. Now, suppose that, in the simplest kind of a common cause pattern, $z$ is a common cause of both $x$ and $y$. Then, the following relations hold for the various kinds of probabilities:

$$
\begin{gathered}
\operatorname{Pr}(x \mid z)>\operatorname{Pr}(x \mid \sim z) \\
\operatorname{Pr}(y \mid z)>\operatorname{Pr}(y \mid \sim z) \\
\operatorname{Pr}(y \mid z \& x)=\operatorname{Pr}(y \mid z \& \sim x) \\
\operatorname{Pr}(y \mid \sim z \& x)=\operatorname{Pr}(y \mid \sim z \& \sim x) \\
\operatorname{Pr}(x \& y \mid z)=\operatorname{Pr}(x \mid z) \operatorname{Pr}(y \mid z) \\
\operatorname{Pr}(x \& y \mid \sim z)=\operatorname{Pr}(x \mid \sim z) \operatorname{Pr}(y \mid \sim z)
\end{gathered}
$$

where $\sim$ denotes the negation. Relations (1) and (2) correspond to the assumption that $z$ is a common cause of $x$ and $y$; (3) and (4) say that the correlation between $x$ and $y$ disappears when $z$ is held fixed (positively or negatively) and (5) as well as (6) mean that when $z$ is held fixed (positively or negatively), the joint probability of $x$ and $y$ will be factorized.

\section{A Local Contextual Hidden-Variable Model}

Let us consider an ideal version of EPR-Bohm experiment in which a spin zero source emits two entangled spin $\frac{1}{2}$ particles in the singlet-state [8]. Alice (Bob) measures the spin component of particle 1 (2) along the direction 
$\widehat{a}(\widehat{b})$, denoted by $S_{a}^{(1)}\left(S_{b}^{(2)}\right)$ which takes the values $r= \pm 1(q= \pm 1)$ in units of $\frac{\hbar}{2}$. The directions $\widehat{a}$ and $\widehat{b}$ lie on the $y$ - $z$ plane. Now, we consider a hidden-variable model in which we have complete information about the spin component of each particle along any direction, given that the corresponding hidden variables along the same direction are known. Moreover, this model enables us to make some statistical predictions along the other directions. The framework of our proposed hidden-variable model consists of the following suppositions:

S1. The set of hidden variables $\lambda_{j,-j}^{n}(\widehat{n}=\widehat{a}, \widehat{b}, \ldots ; j= \pm 1$ in units of $\frac{\hbar}{2}$ ) constitute the field of common cause, where $\widehat{n}=\sin \theta$ $\widehat{y}+\cos \theta \widehat{z}$ is a unit vector in the $y-z$ plane. Taking into account the conservation principle of spin angular momentum, $\lambda_{j,-j}^{n}$ uniquely determine the values $j$ and $-j$ for the spin components of particles 1 and 2 , respectively, along the direction $\widehat{n}$. The outcomes of any spin measurement along $\widehat{n}$ are also assumed to be predetermined by $\lambda_{j,-j}^{n}$.

S2. The spin component $S_{\lambda_{j,-j}^{n}}^{(i)}= \pm 1$ is assumed to contain complete information about the spin value of particle $i(i=1$ or 2 ) along the direction $\widehat{n}$. Subsequently, we define a spin vector along $\widehat{n}$ which is a unit vector, i.e.,

$$
\overrightarrow{S_{\lambda_{j,-j}^{n}}^{(1)}}=j \widehat{n} \text { and } \overrightarrow{S_{\lambda_{j,-j}^{n}}^{(2)}}=-j \widehat{n}
$$

S3. There is an equal chance for the hidden variables $\lambda_{+-}^{n}$ and $\lambda_{-+}^{n}$ to occur in a given run of the experiment. So, we are assuming that:

$$
\operatorname{Pr}\left(\lambda_{+-}^{n}\right)=\operatorname{Pr}\left(\lambda_{-+}^{n}\right)=\frac{1}{2}
$$

where Pr stands for probability.

S4. Regarding the stochastic character of the model, if $\lambda_{j,-j}^{n}$ are given, we shall have only information about the mean value of the spin component of particle $i$ along another direction $\widehat{n^{\prime}} \neq \widehat{n}$, which is denoted by $E^{(i)}\left(\lambda_{j,-j}^{n}, \widehat{n^{\prime}}\right)$. The mean value is postulated to be obtained by projecting the spin vector $\overrightarrow{S_{\lambda_{j,-j}^{n}}^{(i)}}$ on the purposed direction. That is,

$$
E^{(i)}\left(\lambda_{j,-j}^{n}, \widehat{n^{\prime}}\right)=\overrightarrow{S_{\lambda_{j,-j}^{(i)}}^{(i)}} \widehat{n^{\prime}}
$$


Applying $\mathbf{S} 4$ for $\widehat{n}=\widehat{n^{\prime}}$, one gets

$$
E^{(1)}\left(\lambda_{j,-j}^{n}, \widehat{n}\right)=j, E^{(2)}\left(\lambda_{j,-j}^{n}, \widehat{n}\right)=-j ;
$$

which means that we have complete information along the direction $\widehat{n}$, given that $\lambda_{j,-j}^{n}$ is known. Furthermore, Using $\mathbf{S} 2$ and $\mathbf{S 4}$, one can also show that, e.g.,

$$
\begin{aligned}
E^{(2)}\left(\lambda_{j,-j}^{n}, \widehat{n^{\prime}}\right) & =\overrightarrow{S_{\lambda_{j,-j}^{n}}^{(2)}} \cdot \widehat{n^{\prime}}=-j \widehat{n} \cdot \widehat{n^{\prime}} \\
& =-j \cos \varphi_{n n^{\prime}}
\end{aligned}
$$

where $\varphi_{n n^{\prime}}=\left|\widehat{n}-\widehat{n^{\prime}}\right|$. Similarly, $E^{(1)}\left(\lambda_{j,-j}^{n}, \widehat{n^{\prime}}\right)=j \cos \varphi_{n n^{\prime}}$.

Considering S1 and S2, when Alice measures the spin component of the first particle along $\widehat{a}$ and obtains a result $r$, it will be obvious for her that the hidden variables $\lambda_{r,-r}^{a}$ have caused such a result. She can also infer that if Bob measures along the same direction, he will obtain the result $-r$. However, Alice cannot predict with certainty that if Bob measures along a different direction $\widehat{b} \neq \widehat{a}$, what result he will obtain. Nevertheless, using the assumptions $\mathbf{S 1}, \mathbf{S} 2$ and $\mathbf{S} 4$, after her measurement, Alice can refer to $\lambda_{r,-r}^{a}$ to have some statistical predictions at a subquantum level. For example, according to the relation (11) she can predict that the mean value of Bob's results will be $E^{(2)}\left(\lambda_{r,-r}^{a}, \widehat{b}\right)=-r \cos \varphi_{a b}$, if he measures along $\widehat{b}^{1}$. Albeit this result is not observable, one should average over the hidden variables $\lambda_{r,-r}^{a}$ to obtain the empirical results (see below).

The same situation holds for Bob: After his measurement along $\widehat{b}$ and getting the result $q$, he concludes that the mean value of Alice's results will be definitely $E^{(1)}\left(\lambda_{-q, q}^{b}, \widehat{a}\right)=-q \cos \varphi_{a b}$. (For $\widehat{a}=\widehat{b}, r=-q$, according to S1.) Since the choice of measuring direction can be arbitrarily made by each local observer, different sets of hidden variables may be used by Alice and Bob to make predictions about the results of the other one. These predictions might be different at a subquantum level. The hidden variables, however, constitute the same field of common cause. So, each observer can refer to a definite set of hidden variables to reproduce the observational predictions. Their predictions should be the same, experimentally. Hence, to reproduce the Bell correlations, Alice can use the local hidden variables $\lambda_{r,-r}^{a}$, when she reveals the result $r$ for $S_{a}^{(1)}$, which we call hereafter the Alice description. Bob can also invoke $\lambda_{-q, q}^{b}$, when he reveals the result $q$ for $S_{b}^{(2)}$, which we call the

\footnotetext{
${ }^{1}$ Indeed, this is only the analytical form of the prediction, not its numerical one. To have knowledge of the latter case, one needs an exact knowledge of $\varphi_{a b}$ too.
} 
Bob description (Fig 1.) As we shall show in the following, the observational results in both descriptions are the same and they are supposed to be equal to the predictions of quantum mechanics. In the following, we first use the Alice description.

To see some of the predictions of this model at the level of hidden variables, it is apparent from $\mathbf{S 1}$ and $\mathbf{S 2}$ that:

$$
\operatorname{Pr}\left(S_{a}^{(1)}=r, S_{a}^{(2)}=-r \mid \lambda_{r,-r}^{a}, \widehat{a}, \widehat{a}\right)=1 ; \operatorname{Pr}\left(S_{a}^{(1)}=r, S_{a}^{(2)}=r \mid \lambda_{r,-r}^{a}, \widehat{a}, \widehat{a}\right)=0
$$

Then, due to the factorizability of the joint probabilities in (5), in the presence of the common cause (supposing in $\mathbf{S 1}$ ), one can show that

$$
\operatorname{Pr}\left(S_{a}^{(1)}=r, S_{b}^{(2)}=q \mid \lambda_{r,-r}^{a}, \widehat{a}, \widehat{b}\right)=\operatorname{Pr}\left(S_{a}^{(1)}=r \mid \lambda_{r,-r}^{a}, \widehat{a}\right) \operatorname{Pr}\left(S_{b}^{(2)}=q \mid \lambda_{r,-r}^{a}, \widehat{b}\right)
$$

Here, $\operatorname{Pr}\left(S_{a}^{(1)}=r \mid \lambda_{r,-r}^{a}, \widehat{a}\right)=1$ and

$$
\operatorname{Pr}\left(S_{b}^{(2)}=q \mid \lambda_{r,-r}^{a}, \widehat{b}\right)=\frac{1}{2}\left[1+q E^{(2)}\left(\lambda_{r,-r}^{a}, \widehat{b}\right)\right]=\frac{1}{2}\left[1-r q \cos \varphi_{a b}\right]
$$

where the last equality is obtained by using the relation (11).

We can also calculate the correlation function both at the level of hiddenvariables and quantum mechanics. The correlation function at the level of hidden variables is equal to:

$$
C_{\lambda_{r,-r}^{a}}\left(S_{a}^{(1)}, S_{b}^{(2)}\right)=E^{(12)}\left(\lambda_{r,-r}^{a}, \widehat{a}, \widehat{b}\right)-E^{(1)}\left(\lambda_{r,-r}^{a}, \widehat{a}\right) E^{(2)}\left(\lambda_{r,-r}^{a}, \widehat{b}\right)
$$

where $E^{(12)}\left(\lambda_{r,-r}^{a}, \widehat{a}, \widehat{b}\right)$ is the average value of the spin components of the two particles along $\widehat{a}$ and $\widehat{b}$, and it is defined as:

$$
E^{(12)}\left(\lambda_{r,-r}^{a}, \widehat{a}, \widehat{b}\right)=\sum_{k, l= \pm 1} k l \operatorname{Pr}\left(S_{a}^{(1)}=k, S_{b}^{(2)}=l \mid \lambda_{r,-r}^{a}, \widehat{a}, \widehat{b}\right)
$$

Using the relations (13) and (14) in (16), one gets:

$$
E^{(12)}\left(\lambda_{+-}^{a}, \widehat{a}, \widehat{b}\right)=E^{(12)}\left(\lambda_{-+}^{a}, \widehat{a}, \widehat{b}\right)=-\cos \varphi_{a b}
$$

Accordingly, we reach the following result:

$$
C_{\lambda_{r,-r}^{a}}\left(S_{a}^{(1)}, S_{b}^{(2)}\right)=-\cos \varphi_{a b}-r\left(-r \cos \varphi_{a b}\right)=0
$$


which means that there is no correlation at the subquantum level, as was expected.

In quantum mechanics, the correlation function is equal to:

$$
C_{Q M}\left(S_{a}^{(1)}, S_{b}^{(2)}\right)=\left\langle S_{a}^{(1)} S_{b}^{(2)}\right\rangle-\left\langle S_{a}^{(1)}\right\rangle\left\langle S_{b}^{(2)}\right\rangle
$$

where $\langle\ldots\rangle$ stands for the quantum mechanical expectation values. Using S3, one can show that

$$
\begin{aligned}
\left\langle S_{b}^{(2)}\right\rangle & =\operatorname{Pr}\left(\lambda_{+-}^{a}\right) E^{(2)}\left(\lambda_{+-}^{a}, \widehat{b}\right)+\operatorname{Pr}\left(\lambda_{-+}^{a}\right) E^{(2)}\left(\lambda_{-+}^{a}, \widehat{b}\right) \\
& =\frac{1}{2}\left(-r \cos \varphi_{a b}\right)+\frac{1}{2}\left(+r \cos \varphi_{a b}\right)=0
\end{aligned}
$$

where we have used the relation (11) for $\widehat{n}=\widehat{a}$ and $\widehat{n^{\prime}}=\widehat{b}$. Similarly one can show that $\left\langle S_{a}^{(1)}\right\rangle=0$. We also have

$$
\begin{aligned}
\left\langle S_{a}^{(1)} S_{b}^{(2)}\right\rangle & =\operatorname{Pr}\left(\lambda_{+-}^{a}\right) E^{(12)}\left(\lambda_{+-}^{a}, \widehat{a}, \widehat{b}\right)+\operatorname{Pr}\left(\lambda_{-+}^{a}\right) E^{(12)}\left(\lambda_{-+}^{a}, \widehat{a}, \widehat{b}\right) \\
& =\frac{1}{2}\left(-\cos \varphi_{a b}\right)+\frac{1}{2}\left(-\cos \varphi_{a b}\right)=-\cos \varphi_{a b}
\end{aligned}
$$

in which the relation (17) is used. Relations (20) and (21) lead us to the following result:

$$
C_{Q M}\left(S_{a}^{(1)}, S_{b}^{(2)}\right)=-\cos \varphi_{a b}
$$

which is the correlation function predicted by quantum mechanics.

The same results could be obtained, if we used the Bob description. For example, one could replace the left hand side of relation (14) with $\operatorname{Pr}\left(S_{a}^{(1)}=r \mid \lambda_{-q, q}^{b}, \widehat{a}\right)$ and get the same result as $\frac{1}{2}\left[1-r q \cos \varphi_{a b}\right]$. Here, $\operatorname{Pr}\left(S_{b}^{(2)}=q \mid \lambda_{-q, q}^{b}, \widehat{b}\right)=1$ and the relations (17) to (22) could be derived again, if one substitutes $\lambda_{-q, q}^{b}$ in place of $\lambda_{r,-r}^{a}$. This shows that each local observer by herself (himself) can simulate Bell correlations without any communication with her (his) distant partner. So, there is no communication cost for simulating Bell correlations in our approach [4]. This demonstrates the correct application of a common cause description in an appropriate manner.

\section{Locality versus Contextuality}

Our proposed model is an instance of a local contextual hidden-variable theory. Contextulaity in our definition means that we should always refer to a definite set of hidden variables, in a given measuring setup, to be 
able to have some observational predictions. Hence, the joint probability of $S_{a}^{(1)}$ and $S_{b}^{(2)}$ being $r$ and $q$ respectively, in a context which is characterized by the hidden variables $\lambda_{r,-r}^{a}$ and the measuring directions $\widehat{a}$ and $\widehat{b}$ in Alice description (as was denoted by $\operatorname{Pr}\left(S_{a}^{(1)}=r, S_{b}^{(2)}=q \mid \lambda_{r,-r}^{a}, \widehat{a}, \widehat{b}\right)$ in (13)), is essentially different from $\operatorname{Pr}\left(S_{a}^{(1)}=r, S_{b}^{(2)}=q \mid \lambda_{-r, r}^{a}, \widehat{a}, \widehat{b}\right)$ and/or $\operatorname{Pr}\left(S_{a^{\prime}}^{(1)}=r, S_{b}^{(2)}=q \mid \lambda_{r,-r}^{a^{\prime}}, \widehat{a^{\prime}}, \widehat{b}\right)$ where in the latter case, Alice measures the spin component of the first particle along a different direction $\widehat{a^{\prime}} \neq \widehat{a}$. We are not allowed to sum up conditional probabilities in different contexts where distinctive hidden variables characterizes different conditionals. Consequently, Bell's inequality cannot be derived because different sets of hidden variables (including different pieces of information) should be used in different contexts.

However, the model is obviously local. Alice and Bob refer to their own local hidden variables $\lambda_{r,-r}^{a}$ and $\lambda_{-q, q}^{b}$, respectively. There is no causal link between them. Each local observer refers to her (his) context dependent hidden variables to make a proper prediction. Notwithstanding, the predictions of distant observers are not necessarily the same, whenever a hidden-variable description is taken into account. They have no correlation at this level. For example, Alice can attribute the probability $\operatorname{Pr}\left(S_{a}^{(1)}=r \mid \lambda_{r,-r}^{a}, \widehat{a}\right)=1$ to the outcome $S_{a}^{(1)}=r$, whereas Bob predicts $\operatorname{Pr}\left(S_{a}^{(1)}=r \mid \lambda_{-q, q}^{b}, \widehat{a}\right)=$ $\frac{1}{2}\left[1-r q \cos \varphi_{a b}\right]$ for the same outcome at the same time. Nevertheless, their observational predictions are always the same. The spurious correlation appears when the local hidden variables are statistically averaged.

It is also important to stress here that the statistical description of this model is intrinsically realistic. While in quantum mechanics probabilities do not describe the current situation of the system, here we can describe the statistical behavior of the particles in a definite context, if the corresponding hidden variables are known. For example, we can simultaneously attribute probabilities to the spin components of particle 2 along different directions. That is, all spin components are real and the relation (14) holds true for every $\widehat{b}$, given that $\lambda_{r,-r}^{a}$ are known at the same time. But in practice, since one cannot measure $S_{b}^{(2)}$ for different directions simultaneously, we should use the counterfactual statements for what could be described for different directions in a given measuring setup. This means that the observational predictions of this model for incompatible observables should be interpreted counterfactually. This is different from the standard interpretation of quantum mechanics in which the randomness of events is usually believed to be irreducible. Instead, the quantum results are always reproduced in this model, because we are ignorant of which of the hidden variables, e.g., $\lambda_{+-}^{a}$ or $\lambda_{-+}^{a}$ is applicable 
in different runs of a given experiment.

\section{A Classical example}

We are now going to present a classical counterpart for a Bell-type experiment which obeys similar rules as what was mentioned for a local-contextual model in section 3. Let us assume that Alice and Bob are two observers who are located at different positions with a space-like separation. There is no communication between them. Intermediately, there is a place where a sender (Sam) stores balls with different colors. On each ball, one of the two signs + or - is inscribed. Sam sends balls with different colors to each of the two observers in different stages of an experiment successively. Each observer has a color-recognizer device which is able to detect the passage of any colored ball but can be adjusted to identify only one type of color during each step of an experiment. Choosing from Sam's colors set, let us assume here that Alice decides to collect balls with color $a$ and Bob collects balls with color $b$, standing for, e.g., amber and blue, respectively. They are not aware of which color their partner chooses, nor Sam needs having knowledge of their decision. Subsequently, Alice and Bob record the signs on their balls. Then, they will upload their outcomes to a remote computer which can calculate the correlation of data.

As a matter of fact, this experiment is composed of different stages. Without loss of generality, one can suppose that Sam owns a set of three colored balls including amber $(a)$, blue $(b)$ and cherry $(c)$. In three different stages, Sam sends both members of the pairs $(a, b),(a, c)$ and $(c, b)$ to Alice and Bob. For each pair of colored balls including $(a, b),(a, c)$ and $(c, b)$, Sam exploits specific executive algorithms which cause definite correlations between the signs inscribed on balls. In each stage of the experiment, Alice's (Bob's) device is capable of recognizing only one of the two colors $a$ or $c$ ( $b$ or $c$ ). The reader can find a full description of our proposed experiment in the following Appendix. Nevertheless, for our present purposes, let us consider a more restricted situation where Alice and Bob choose $a$ and $b$ colors, respectively. So, it is sufficient henceforth to focus on those events in which only $a$ and $b$ colored balls are detected simultaneously, and leave out the $c$ events. In this way, Sam is committed to send these colors according to two complementary executive algorithms which we call $\mathcal{A}_{1}$ and $\mathcal{A}_{2}$. Each algorithm includes some important features which are described in the following:

1- Sam either sends a colored balls with engraved symbols + (hereafter called as $a_{A}+$ ) or the same colored balls with engraved 
symbols $-\left(a_{A}-\right)$ along with $b$ colored balls (denoted by $\left.b_{A}\right)$ to Alice. The first situation is controlled by $\mathcal{A}_{1}$ algorithm and the second one by $\mathcal{A}_{2}$. Sam uses the two algorithms with equal chances, i.e., $\operatorname{Pr}\left(\mathcal{A}_{1}\right)=\operatorname{Pr}\left(\mathcal{A}_{2}\right)=\frac{1}{2}$.

2- Suppose, for example, that Sam sends an $a_{A}+$ and a $b_{A}-$ ball to Alice. Then, at the same time, he will surely send an $a_{B}-$ and a $b_{B}+$ ball to Bob. This means that Sam takes into account the anticorrelation rule in choosing \pm symbols for the same colored balls which are sent to different observers. The anticorrelation rule is fulfilled by either of the $\mathcal{A}_{1}$ and $\mathcal{A}_{2}$ algorithms. Strictly speaking, e.g., under the $\mathcal{A}_{1}$ algorithm, $a_{A}+$ and $a_{B}-$ balls are always sent to Alice and Bob, respectively. The $b$ colored balls should also satisfy the anticorrelation rule at the same time.

3- When Sam executes the $\mathcal{A}_{1}$ algorithm, the probability that Bob records $b_{B}+$ results is chosen to be 0.15 . This means that Sam sends $b+$ balls to Bob (joined with $a+$ balls to Alice), with a frequency of 15 per $100 b$ colored balls ${ }^{2}$. When Sam uses the $\mathcal{A}_{2}$ algorithm, the probability that Bob records the same outcome is 0.85 (i.e., a frequency of 85 per $100 b$ colored balls). Accordingly, when Alice records $a_{A}+\left(a_{A}-\right)$ results, Bob will receive $b_{B}+$ $\left(b_{B}-\right)$ balls with a probability of 0.15 .

4- When Sam uses the $\mathcal{A}_{1}$ algorithm, each $b_{A}$ - ball is also sent to Alice with a probability of 0.15 . This is a consequence of the anticorrelation rule in 2 . Hence, according to $\mathcal{A}_{2}$ algorithm, the probability that the same ball is sent to Alice is 0.85 . This accomplishes the stochastic characters of two executive algorithms used by Sam.

5- This is an ideal experiment, in the sense that the color-recognizer device is capable of registering any colored ball passing through it, even if it cannot recognize its color. The experiment is ended when Alice and Bob both have full registered data (i.e., the number of all color-registered balls is compatible with all passing balls) for $a$ and $b$ colored balls, respectively.

Table 1 summarizes the executive routines of $\mathcal{A}_{1}$ and $\mathcal{A}_{2}$ in terms of some definite frequencies. Here, e.g., the results $\left(a_{A}+, b_{A}-; b_{B}+, a_{B}-\right)$ denote

\footnotetext{
${ }^{2}$ Here, we are always assuming that the probability of an event is equivalent to the frequency of occurrence of the same event. So, when we say that the probability of a given outcome is, e.g., 0.15 , we mean there are $N$ total results for which our supposed outcome occures $0.15 N$ times.
} 
that Sam sends simultaneously a pair of $a$ colored balls with signs + and - as well as a pair of $b$ colored balls with opposite signs to Alice and Bob, respectively. (Here, we are neglecting the $c$ colored balls, as was said before. For a full description of this experiment see the following Appendix.) Under the $\mathcal{A}_{1}$, the probability (frequency) of occurrence of this event is equal to:

$$
\begin{aligned}
\operatorname{Pr}\left(a_{A}+, b_{A}-; b_{B}+, a_{B}-\mid \mathcal{A}_{1}\right) & =\operatorname{Pr}\left(a_{A}+; b_{B}+\mid \mathcal{A}_{1}\right) \operatorname{Pr}\left(b_{A}-; a_{B}-\mid \mathcal{A}_{1}, a_{A}+, b_{B}+\right) \\
& =\operatorname{Pr}\left(b_{A}-; a_{B}-\mid \mathcal{A}_{1}\right) \operatorname{Pr}\left(a_{A}+; b_{B}+\mid \mathcal{A}_{1}, b_{A}-, a_{B}-\right) \\
& =0.15 \times 1=0.15
\end{aligned}
$$

The frequencies of other results can be defined similarly.

\begin{tabular}{|c|c|c|}
\hline Frequency $\backslash$ Results & $\left(a_{A}+, b_{A}-; b_{B}+, a_{B}-\right)$ & $\left(a_{A}+, b_{A}+; b_{B}-, a_{B}-\right)$ \\
\hline Frequency according to $\mathcal{A}_{1}$ & 0.15 & 0.85 \\
\hline Frequency $\backslash$ Results & $\left(a_{A}-, b_{A}-; b_{B}+, a_{B}+\right)$ & $\left(a_{A}-, b_{A}+; b_{B}-, a_{B}+\right)$ \\
\hline Frequency according to $\mathcal{A}_{2}$ & 0.85 & 0.15 \\
\hline
\end{tabular}

Table 1. The executive routines of $\mathcal{A}_{1}$ and $\mathcal{A}_{2}$ in terms of some given probabilities.

The results indicated in table 1 are not exactly what Alice and Bob can observe. As was said before, Alice only detects one of the two results $a_{A}+$ or $a_{A}-$. Correspondingly, Bob can only record $b_{B}+$ and $b_{B}-$ outcomes. So, what both observers can really observe is that they are receiving their desired colors with an equal distribution of + and - signs. At the end of experiment, Alice and Bob upload their data to a remote computer which is able to compare their results and calculate the correlation of them. Table 2 demonstrates what the remote computer calculates for the frequency of occurrence of the observed results and their correlation.

\begin{tabular}{|c|c|c|c|c|}
\hline Statistical Calculations \Results & $\left(a_{A}+; b_{B}+\right)$ & $\left(a_{A}+; b_{B}-\right)$ & $\left(a_{A}-; b_{B}+\right)$ & $\left(a_{A}-; b_{B}-\right)$ \\
\hline Frequency & $\frac{1}{2} \times 0.15$ & $\frac{1}{2} \times 0.85$ & $\frac{1}{2} \times 0.85$ & $\frac{1}{2} \times 0.15$ \\
\hline Correlation & \multicolumn{5}{|c|}{-0.7} \\
\hline
\end{tabular}

Table 2. The remote computer calculates the frequency of occurrence of detected results and their correlation. 
Here, for instance, the joint probability of getting an $a_{A}+$ result by Alice in conjunction with a $b_{B}+$ result by Bob is equal to:

$$
\begin{aligned}
\operatorname{Pr}\left(a_{A}+; b_{B}+\right) & =\operatorname{Pr}\left(a_{A}+\right) \operatorname{Pr}\left(b_{B}+\mid a_{A}+\right) \\
& =\operatorname{Pr}\left(b_{B}+\right) \operatorname{Pr}\left(a_{A}+\mid b_{B}+\right) \\
& =\frac{1}{2} \times 0.15
\end{aligned}
$$

Considering the outcomes of Alice and Bob collectively, the above result, e.g., can be calculated directly by the remote computer. However, one can also reach the same result, using Sam's information about the statistical features of two executive algorithms $\mathcal{A}_{1}$ and $\mathcal{A}_{2}$ described above:

$$
\begin{aligned}
\operatorname{Pr}\left(a_{A}+; b_{B}+\right) & =\operatorname{Pr}\left(a_{A}+; b_{B}+\mid \mathcal{A}_{1}\right) \operatorname{Pr}\left(\mathcal{A}_{1}\right)+\operatorname{Pr}\left(a_{A}+; b_{B}+\mid \mathcal{A}_{2}\right) \operatorname{Pr}\left(\mathcal{A}_{2}\right) \\
& =0.15 \times \frac{1}{2}+0 \times \frac{1}{2}=0.15 \times \frac{1}{2}
\end{aligned}
$$

Now let us see what Sam obtains for the correlations of the twofold results in table 2 in the presence of the determining factors $\mathcal{A}_{1}$ and $\mathcal{A}_{2}$ which should be viewed as two complementary calculational contexts. Hence, one can define, e.g.:

$$
C_{\mathcal{A}_{1}}\left(a_{A}, b_{B}\right)=\left\langle a_{A} b_{B}\right\rangle_{\mathcal{A}_{1}}-\left\langle a_{A}\right\rangle_{\mathcal{A}_{1}}\left\langle b_{B}\right\rangle_{\mathcal{A}_{1}}
$$

where $C_{\mathcal{A}_{1}}\left(a_{A}, b_{B}\right)$ is the correlation function under the $\mathcal{A}_{1}$ algorithm and the other functions are mean values under the same program. From table 1, it is apparent that $\left\langle a_{A} b_{B}\right\rangle_{\mathcal{A}_{1}}=-0.7,\left\langle a_{A}\right\rangle_{\mathcal{A}_{1}}=+1$ and $\left\langle b_{B}\right\rangle_{\mathcal{A}_{1}}=-0.7$. So, $C_{\mathcal{A}_{1}}\left(a_{A}, b_{B}\right)=0$. One can similarly show that $C_{\mathcal{A}_{2}}\left(a_{A}, b_{B}\right)$ is also equal to zero under the $\mathcal{A}_{2}$ algorithm. Consequently, the correlation disappears when a third variable $\left(\mathcal{A}_{1}\right.$ or $\left.\mathcal{A}_{2}\right)$ is introduced and held fixed. Accordingly, the executive algorithms $\mathcal{A}_{1}$ and $\mathcal{A}_{2}$ constitute a field of common cause.

Comparing this with a Bell-type experiment, if one replaces the colored balls $a$ and $b$ with the spin components of two particles along the directions $\widehat{a}$ and $\widehat{b}$ respectively, it will be apparent that the two executive algorithms $\mathcal{A}_{1}$ and $\mathcal{A}_{2}$ in the present example stand for the hidden variables $\lambda_{+-}^{a}$ and $\lambda_{-+}^{a}$ in our Bell story. Here, however, we restricted ourselves to a single measuring setup which includes only $a$ and $b$ colored balls. So, $\mathcal{A}_{1}$ and $\mathcal{A}_{2}$ are solely defined in such a measuring context. Yet, in such a context too, the two algorithms require complementary calculational conditions under which one is not allowed to sum up the probabilities directly. The expressions like $\operatorname{Pr}\left(\ldots, \ldots \mid \mathcal{A}_{1}\right)+\operatorname{Pr}\left(\ldots, \ldots \mid \mathcal{A}_{2}\right)$ are meaningless. Alternatively, if one applies 
the probability rules correctly, one can hide the role of different conditions. Correlation appears as an emergent result of such a disguisement.

\section{Conclusion}

Bell's theorem states that there are no local hidden variables which can screen off the mysterious correlations of two entangled particles. However, it is still important to know what we can infer about the nature of these correlations. Is there any non-local action? Or, do Alice and Bob only update their state of knowledge, when they receive new information via the classical channels of information? Here, the correlation appears when the whole information is considered. A positive response to either of the above questions introduces other problems which are regularly discussed in the literature $[9,10,11]$.

There is, however, another possibility when one introduces the notion of contextuality. The consistency of the local contextual hidden-variable theories is not necessarily ruled out by Bell's theorem, although this really depends on what one means by the term contextual (for example, see references $[6,12])$. Here, we have tried to construct a local contextual hidden-variable model which can reproduce the predictions of quantum mechanics. The contextual character of this model requires that the type of hidden variables used by two observers be held fixed according to their corresponding measuring setup. So, it is feasible to explain the two-particle quantum correlations according to the common cause principle in a contextual manner.

\section{Appendix}

Let us assume that in three different stages of an experiment- hereafter called first, second and third stages- Sam sends three pairs of colored balls $(a, b),(a, c)$ and $(c, b)$ to both Alice and Bob. Then, after many trials, Alice and Bob upload their registered results in distinct categories (indicating the time and duration of the corresponding experiment) to a remote computer which can perform statistical calculations. Each stage of experiment is controlled by two complementary executive algorithms which are chosen by Sam with equal chance. Hence, there are three equiprobable couples of such executive algorithms: $\left(\mathcal{A}_{1}, \mathcal{A}_{2}\right),\left(\mathcal{A}_{1}^{\prime}, \mathcal{A}_{2}^{\prime}\right)$, and $\left(\mathcal{A}_{1}^{\prime \prime}, \mathcal{A}_{2}^{\prime \prime}\right)$. Either of the two first algorithms $\mathcal{A}_{1}$ or $\mathcal{A}_{2}$ is used when Sam decides to send $\left(a_{A}+, b_{A} \pm\right)$ joined with $\left(b_{B} \mp, a_{B}-\right)$ balls or $\left(a_{A}-, b_{A} \pm\right)$ joined with $\left(b_{B} \mp, a_{B}+\right)$ balls to Alice and Bob respectively, as was described in section 5. The second and the third pairs of algorithms are characterized by replacing $b$ or $a$ colored balls with $c$ s in the above settings, correspondingly. Table $\mathbf{A}_{\mathbf{1}}$ shows the

probabilistic features of two algorithms $\mathcal{A}_{1}^{\prime}$ and $\mathcal{A}_{1}^{\prime \prime}$. It is assumed that for 
both situations, when Sam executes any of the two algorithms $\mathcal{A}_{1}^{\prime}$ or $\mathcal{A}_{1}^{\prime \prime}$, the probability that Bob records a positive result is equal to 0.04 . For simplicity, we have not mentioned the executive routines of $\mathcal{A}_{2}^{\prime}$ and $\mathcal{A}_{2}^{\prime \prime}$ which are simply the complements of two earlier programs, respectively.

\begin{tabular}{|c|c|c|}
\hline Frequency $\backslash$ Results & $\left(a_{A}+, c_{A}-; c_{B}+, a_{B}-\right)$ & $\left(a_{A}+, c_{A}+; c_{B}-, a_{B}-\right)$ \\
\hline Frequency according to $\mathcal{A}_{1}^{\prime}$ & 0.04 & 0.96 \\
\hline Frequency $\backslash$ Results & $\left(c_{A}+, b_{A}-; b_{B}+, c_{B}-\right)$ & $\left(c_{A}+, b_{A}+; b_{B}-, c_{B}-\right)$ \\
\hline Frequency according to $\mathcal{A}_{1}^{\prime \prime}$ & 0.04 & 0.96 \\
\hline
\end{tabular}

Table $\mathbf{A}_{\mathbf{1}}$. The executive routines of $\mathcal{A}_{1}^{\prime}$ and $\mathcal{A}_{1}^{\prime \prime}$ in terms of some given probabilities.

When Sam executes, say, $\mathcal{A}_{1}^{\prime}$ algorithm, it may happen that Bob decides to collect $b$ colored balls instead of $c \mathrm{~s}$ in a given course of experiment. For such a case, he does not record any result. In other words, $\operatorname{Pr}\left(a_{A}+, b_{A} \pm ; b_{B} \mp, a_{B}-\right.$ | $\left.\mathcal{A}_{1}^{\prime}\right)=0$. A similar situation may happen for Alice. So, in some given courses, it may occur that Alice and/or Bob, because of their unsuitable choices, register no result. Nevertheless, the experiment is repeated for many times and one can assume that there are always situations where Alice and Bob register simultaneously all transmitted colored balls in a given course. Moreover, because in all executive algorithms, the probability of occurring an event is supposed to be equivalent to the frequency of occurrence of the same event (see footnote in section 5), a remote computer can neglect the stages in which any of the two observers detects no result and calculate the frequency of occurrence of the joint outcomes in those stages where all joint data are registered. This can be done by comparing the total sum of color-registered data with all that the color-recognizer device detects as passing balls. The experiment is declared to be terminated when Alice and Bob both have full registered data, i.e., when all color-registered balls match the passing balls for all triple stages (see assumption 5 in section 5 ).

\begin{tabular}{|c|c|c|c|c|}
\hline Statistical Calculations $\backslash$ Results & $\left(a_{A}+; c_{B}+\right)$ & $\left(a_{A}+; c_{B}-\right)$ & $\left(a_{A}-; c_{B}+\right)$ & $\left(a_{A}-; c_{B}-\right)$ \\
\hline Frequency for the second stage & $\frac{1}{2} \times 0.04$ & $\frac{1}{2} \times 0.96$ & $\frac{1}{2} \times 0.96$ & $\frac{1}{2} \times 0.04$ \\
\hline Statistical Calculations $\backslash$ Results & $\left(c_{A}+; b_{B}+\right)$ & $\left(c_{A}+; b_{B}-\right)$ & $\left(c_{A}-; b_{B}+\right)$ & $\left(c_{A}-; b_{B}-\right)$ \\
\hline Frequency for the third stage & $\frac{1}{2} \times 0.04$ & $\frac{1}{2} \times 0.96$ & $\frac{1}{2} \times 0.96$ & $\frac{1}{2} \times 0.04$ \\
\hline
\end{tabular}

Table $\mathbf{A}_{\mathbf{2}}$. The remote computer calculates the frequency of occurrence of a joint result for the second and third stages. 
For the second and third stages of this trilogy, the statistical calculations of the remote computer are summarized in table $\mathbf{A}_{\mathbf{2}}$. Here, all the complementary algorithm pairs $\left(\mathcal{A}_{1}, \mathcal{A}_{2}\right),\left(\mathcal{A}_{1}^{\prime}, \mathcal{A}_{2}^{\prime}\right)$ and $\left(\mathcal{A}_{1}^{\prime \prime}, \mathcal{A}_{2}^{\prime \prime}\right)$ constitute the field of common cause. This means that the corresponding correlations disappear when any of the two algorithms in each pair is held fixed. On the other hand, each of the above pairs is defined in a specific context which is characterized by a definite pair of colored balls sent to the observers. Disregarding the role of conditional contexts in the application of the probability rules, one can derive some Bell-type inequalities, such as (see, e.g., reference [13])

$$
\operatorname{Pr}\left(a_{A}+; b_{B}+\right) \leq \operatorname{Pr}\left(a_{A}+; c_{B}+\right)+\operatorname{Pr}\left(c_{A}+; b_{B}+\right)
$$

Putting the corresponding results from the tables 2 and $\mathbf{A}_{\mathbf{2}}$ into the above inequality, we shall observe a clear inconsistency:

$$
\frac{1}{2} \times 0.15 \leq \frac{1}{2} \times 0.04+\frac{1}{2} \times 0.04 \text { Or } 0.15 \leq 0.08
$$

The inconsistency, however, is removed, when one remembers that the probability functions in relation (A-1) are derived under different contexts. One such derivation is shown in the relation (25). Indeed, if we apply the

probability rules correctly, the Bell-type inequality (A-1) will be never obtained.

\section{References}

[1] J. S. Bell, Physics 1, 195 (1964).

[2] J. F. Clauser and A. Shimony, Rep. Prog. Phys., 41, 1881 (1978).

[3] J. T. Cushing, in Philosophical Consequences of Quantum Theory, Reflections on Bell's Theorem, edited by J. T. Cushing and E. McMullin (University of Notre Dame Press, Notre Dame, 1989).

[4] B. F. Toner and D. Bacon, Phys. Rev. Lett. 91, 187904 (2003).

[5] L. E. Szabó, Found. Phys. Lett., 8, 421 (1995); Int. J. Theor. Phys., 39, 901 (2000); Int. J. of Theor. Phys., 37, 449 (1998).

[6] W. M. de Muynck, Found. Phys. 34, 717 (2004).

[7] E. Eells, Probabilistic Causality, ( Cambridge University Press, Cambridge,1991), p. 59. 
[8] D. Bohm, Quantum Theory (Prentice-Hall, Englewood Cliffs, NJ, 1951), pp.614-619.

[9] M. Ferrero, Found. Phys. 33, 665 (2003).

[10] A. Plotnitsky, Found. Phys. 33, 1649 (2003).

[11] R. B. Griffiths, Found. Phys. 33, 1423 (2003).

[12] A. Khrennikov and I. Volovich, http://arxiv.org/quant-ph/0211078, http://arxiv.org/quant-ph/0212127.

[13] J. S. Townsend, A Modern Approach To Quantum Mechanics, (University Science Books, Sausalito, 2000), p. 139. 
Figure 1: After a measurement along $\widehat{a}(\widehat{b})$ and getting the result $r(q)$, Alice (Bob) will recognize that the hidden variables $\lambda_{r,-r}^{a}\left(\lambda_{-q, q}^{b}\right)$ have caused the corresponding result for $s_{a}^{(1)}\left(s_{b}^{(2)}\right)$. The hidden variables $\lambda_{j,-j}^{n}(\widehat{n}=\widehat{a}, \widehat{b}, \ldots$; $j= \pm 1$ ) constitute the field of common cause where $\lambda_{r,-r}^{a}$ and $\lambda_{-q, q}^{b}$ stem from. 
This figure "FigCommCause.jpg" is available in "jpg" format from: http://arxiv.org/ps/quant-ph/0504121v1 